\title{
Second Atom-Bond Connectivity Index of Special Chemical Molecular Structures
}

\author{
Wei Gao ${ }^{1}$ and Weifan Wang ${ }^{2}$ \\ ${ }^{1}$ School of Information Science and Technology, Yunnan Normal University, Kunming 650500, China \\ ${ }^{2}$ Department of Mathematics, Zhejiang Normal University, Jinhua 321004, China
}

Correspondence should be addressed to Wei Gao; gaowei@ynnu.edu.cn

Received 7 July 2014; Accepted 27 August 2014; Published 15 October 2014

Academic Editor: Demeter Tzeli

Copyright ( 2014 W. Gao and W. Wang. This is an open access article distributed under the Creative Commons Attribution License, which permits unrestricted use, distribution, and reproduction in any medium, provided the original work is properly cited.

In theoretical chemistry, the second atom-bond connectivity index was introduced to measure the stability of alkanes and the strain energy of cycloalkanes. In this paper, we determine the second atom-bond connectivity index of unilateral polyomino chain and unilateral hexagonal chain. Furthermore, the second $A B C$ indices of $\mathrm{V}$-phenylenic nanotubes and nanotori are presented.

\section{Introduction}

One of the most important applications of chemical graph theory is to measure chemical, physical, and pharmaceutical properties of molecules called alkanes. Several indices that relied on the graphical structure of the alkanes are defined and employed to model both the melting point and boiling point of the molecules. Molecular graph is a topological representation of a molecule such that each vertex represents an atom of molecule, and covalent bonds between atoms are represented by edges between the corresponding vertices.

Specifically, topological index can be regarded as a score function $f: G \rightarrow \mathbb{R}^{+}$, with this property that $f\left(G_{1}\right)=f\left(G_{2}\right)$ if two molecular graphs $G_{1}$ and $G_{2}$ are isomorphic. There are several vertex distance-based and degree-based indices which are introduced to analyze the chemical properties of molecule graph, for instance, Wiener index, PI index, Szeged index, and atom-bond connectivity index. Several papers contributed to determining the indices of special molecular graphs (see Yan et al. [1, 2], Gao and Shi [3], Xi and Gao [4], and Dou et al. [5] for more detail).

All (molecular) graphs considered in this paper are finite, loopless, and without multiple edges. Let $G$ be a (molecular) graph with vertex set $V(G)$ and edge set $E(G)$. All graph notations and terminologies used but undefined in this paper can be found in [6].
The atom-bond connectivity index (shortly, $A B C$ index) is defined by Estrada et al. [7] as

$$
A B C(G)=\sum_{u v \in E(G)} \sqrt{\frac{d(u)+d(v)-2}{d(u) d(v)}} .
$$

Das et al. [8] characterized the molecular graphs as extremal with regard to $A B C$ index. Furtula et al. [9] found the chemical trees with extremal $A B C$ index. Vassilev and Huntington [10] identified certain classes of edges that are important and occur frequently in chemical trees and continuously studied the chemical trees with extremal $A B C$ index in terms of learning how the removal of a certain edge takes place. Chen et al. [11] obtained the atom-bond connectivity index of the zig-zag chain polyomino molecular graphs. Furthermore, they determined the sharp upper bound on the atom-bond connectivity index of catacon densed polyomino molecular graphs with squares and determined the corresponding extremal molecular graphs.

Recently, Graovac and Ghorbani [12] defined a new version of the atom-bond connectivity index, that is, the second atom-bond connectivity index (shortly, second $A B C$ index):

$$
A B C_{2}(G)=\sum_{u v \in(G)} \sqrt{\frac{n(u)+n(v)-2}{n(u) n(v)}},
$$




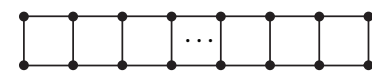

Linear polyomino chain

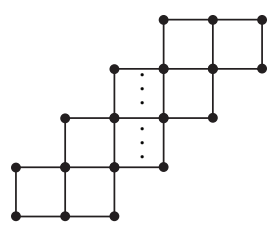

Zig-zag polyomino chain

FIGURE 1: The structure of $L_{n}^{4}$ and $Z_{n}^{4}$.

where, for each $u v \in E(G), n(u)$ is the number of vertices closer to vertex $u$ than vertex $v$ and $n(v)$ defines similarly. In the chemistry applications, second $A B C$ index is used to model both the boiling point and melting point of the molecules. Hence, it is also applied to the pharmaceutical field. Rostami et al. [13] calculated some upper bounds for the second atom-bond connectivity index.

Although there have been several advances in atom-bond connectivity index of molecular graphs, the study of second atom-bond connectivity index of special chemical structures has been largely limited. In addition, as widespread and critical chemical structures, polyomino system, hexagonal system, V-phenylenic nanotubes, and nanotori are widely used in medical science and pharmaceutical field. As an example, polyomino chain is one of the basic chemical structures and exists widely in benzene and alkali molecular structures. For these reasons, we have attracted tremendous academic and industrial interests to research the second atom-bond connectivity of these molecular structures from a mathematical point of view.

The contribution of our paper is threefold. First, we compute the second atom-bond connectivity index of unilateral polyomino chain. Then, the second atom-bond connectivity index of unilateral hexagonal chain is calculated. At last, we derive the second atom-bond connectivity index of Vphenylenic nanotubes and nanotori.

\section{Second Atom-Bond Connectivity Index of Polyomino Chain}

From the view of graph theory, polymino is a finite 2connected planar graph and each interior face is surrounded by a square with length 4 . Polyomino chain is one class of polyomino such that the connection of centres for adjacent squares constitutes a path $c_{1} c_{2} \cdots c_{n}$, where $c_{i}$ is the centre of $i$ th square. Polyomino chain $H_{n}^{4}$ is called a linear chain if the subgraph induced by all 3 -degree vertices is a graph with $n-2$ squares. Furthermore, polyomino chain $H_{n}^{4}$ is called a zigzag chain if the subgraph induced by all vertices with degree $>2$ is path with $n-1$ edges. In what follows, we use $L_{n}^{4}$ and $Z_{n}^{4}$ to denote linear polyomino chain and zig-zag polyomino chain, respectively. For the structure of $L_{n}^{4}$ and $Z_{n}^{4}$, refer to Figure 1.

Use the similar technology raised in Gutman and Klavžar [14], and we define elementary cut as follows. Choose an edge $e$ of the polyomino system and draw a straight line through the center of $e$, orthogonal on $e$. This line will intersect the perimeter in two end points, $P_{1}$ and $P_{2}$. The straight line segment $C$ whose end points are $P_{1}$ and $P_{2}$ is the elementary cut, intersecting the edge $e$. A fragment $S$ in polyomino chain is just maximal linear chain which includes the squares in start and end vertices. Let $l(S)$ be the length of fragment which denotes the number of squares it contained. Let $H_{n}^{4}$ be a polyomino chain with $n$ squares consisting of fragment sequence $S_{1}, S_{2}, \ldots, S_{m}(m \geq 1)$. Denote $l\left(S_{i}\right)=l_{i}(i=$ $1, \ldots, m)$. It is not difficult to verify that $l_{1}+l_{2}+\cdots+l_{m}=$ $n+m-1$ and $\left|V\left(H_{n}^{4}\right)\right|=2 n+2,\left|E\left(H_{n}^{4}\right)\right|=3 n+1$. For the $k$ th fragment of polyomino chain, the cut of this fragment is the cut which intersects with $l_{k}+1$ parallel edges of squares in this fragment. A fragment is called horizontal fragment if its cut parallels the horizontal direction and called vertical fragment if its cut parallels the vertical direction. Unilateral polyomino chain is a special kind of polyomino chain such that, for each vertical fragment, two horizontal fragments (if exists) are adjacent and it appears in the left and right sides, respectively.

Our main result in this section stated as follows presents the second atom-bond connectivity index of unilateral polyomino chain.

Theorem 1. Let $H_{n}^{4}$ be a unilateral polyomino chain consisting of $m$ fragment $S_{1}, S_{2}, \ldots, S_{m}(m \geq 1)$, and let $l\left(S_{i}\right)=l_{i}(i=$ $1, \ldots, m)$ be the length of each fragment. Then, one has

$$
\begin{aligned}
& A B C_{2}\left(H_{n}^{4}\right) \\
& =2 \sum_{j=1}^{l_{1}-1} \sqrt{\frac{n}{j\left(2 \sum_{i=2}^{m} l_{i}-2 m+2\left(l_{1}-j\right)+4\right)}} \\
& +2 \sum_{j=2}^{l_{m}} \sqrt{\frac{n}{\left(2 \sum_{i=1}^{m-1} l_{i}-2 m+2 j+2\right)\left(l_{m}-j+1\right)}} \\
& +2 \sum_{k=2}^{m-1} \sum_{j=2}^{l_{k}-1}\left(n \left(\left(2 \sum_{i=1}^{k-1} l_{i}-2 k+2 j+2\right)\right.\right. \\
& \left.\left.\quad \times\left(\sum_{i=k+1}^{m} l_{i}-(m-k)+\left(l_{k}-j\right)+1\right)\right)^{-1}\right)^{1 / 2}
\end{aligned}
$$




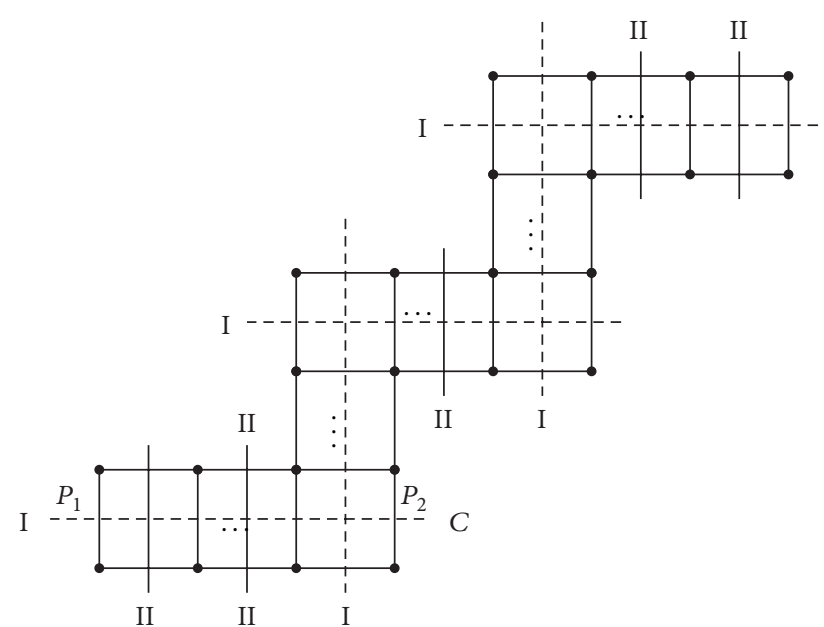

FIGURE 2: I-type cut and II-type cut of unilateral polyomino chain.

$$
\begin{aligned}
&+\sum_{k=1}^{m}\left(l_{k}+1\right) \\
& \times\left(2 n \left(\left(2 \sum_{i=1}^{k-1} l_{i}-2 k+l_{k}+3\right)\right.\right. \\
&\left.\left.\quad \times\left(2 \sum_{i=k+1}^{m} l_{i}-2(m-k)+l_{k}+1\right)\right)^{-1}\right)^{1 / 2} .
\end{aligned}
$$

Proof. The cuts in $H_{n}^{4}$ are divided into two types: I-type and II-type (see Figure 2). An edge is called I-type (or II-type) if it intersects with I-type (or II-type) cut. Now, we consider the following two cases.

Case 1 . If edge $e$ is I-type in $j$ th square of $k$ th fragment (i.e., $e$ is edge which is passed by dotted line in Figure 2), we observe that there is $l_{k}+1$ such edges in $k$ th fragment.

Subcase 1.1. If $k=1$, then we have

$$
\begin{gathered}
n_{1}(e)=l_{1}+1, \\
n_{2}(e)=2 \sum_{i=2}^{m} l_{i}-2 m+l_{1}+3 .
\end{gathered}
$$

Subcase 1.2. If $k=m$, then we obtain

$$
\begin{gathered}
n_{1}(e)=2 \sum_{i=1}^{m-1} l_{i}-2 m+l_{m}+3 \\
n_{2}(e)=l_{m}+1
\end{gathered}
$$

Subcase 1.3. If $2 \leq k \leq m-1$, then we get

$$
\begin{gathered}
n_{1}(e)=2 \sum_{i=1}^{k-1} l_{i}-2 k+l_{k}+3, \\
n_{2}(e)=2 \sum_{i=k+1}^{m} l_{i}-2(m-k)+l_{k}+1 .
\end{gathered}
$$

Case 2. If edge $e$ is II-type in $j$ th square of $k$ th fragment. (i.e., $e$ is the edge which is passed by real line in Figure 2).

Subcase 2.1. If $k=1$, then we yield

$$
\begin{gathered}
n_{1}(e)=2 j \\
n_{2}(e)=2 \sum_{i=2}^{m} l_{i}-2 m+2\left(l_{1}-j\right)+4 .
\end{gathered}
$$

Subcase 2.2. If $k=m$, then we infer

$$
\begin{gathered}
n_{1}(e)=2 \sum_{i=1}^{m-1} l_{i}-2 m+2 j+2, \\
n_{2}(e)=2 l_{m}-2 j+2 .
\end{gathered}
$$

Subcase 2.3. If $2 \leq k \leq m-1$, then we deduce

$$
\begin{gathered}
n_{1}(e)=2 \sum_{i=1}^{k-1} l_{i}-2 k+2 j+2, \\
n_{2}(e)=2 \sum_{i=k+1}^{m} l_{i}-2(m-k)+2\left(l_{k}-j\right)+2 .
\end{gathered}
$$

Hence, the desired conclusion is obtained by combining the above case and the definition of the second atom-bond connectivity index.

Corollary 2. Let $L_{n}^{4}$ be the linear chain with $n$ squares. Then, one has

$$
A B C_{2}\left(L_{n}^{4}\right)=2 \sum_{j=1}^{n-1} \sqrt{\frac{n}{2 j(n-j+1)}}+\sqrt{2 n} .
$$

Proof. By the definition of linear chain, we have $m=1, l_{1}=$ $n, l_{2}=\cdots=l_{m}=0$. In terms of Theorem 1 , we immediately get the result.

Corollary 3. Let $Z_{n}^{4}$ be the zig-zag chain with $n$ squares. Then, one has

$$
A B C_{2}\left(Z_{n}^{4}\right)=4 \sqrt{\frac{1}{2}}+\sum_{k=1}^{m} 3 \sqrt{\frac{2 n}{(2 k+1)(2 n-2 k+1)}}
$$

Proof. By the virtue of the definition of the zig-zag chain, we have $m=n-1$ and $l_{1}=l_{2}=\cdots=l_{m}=2$. In view of Theorem 1 , the result is immediately obtained. 

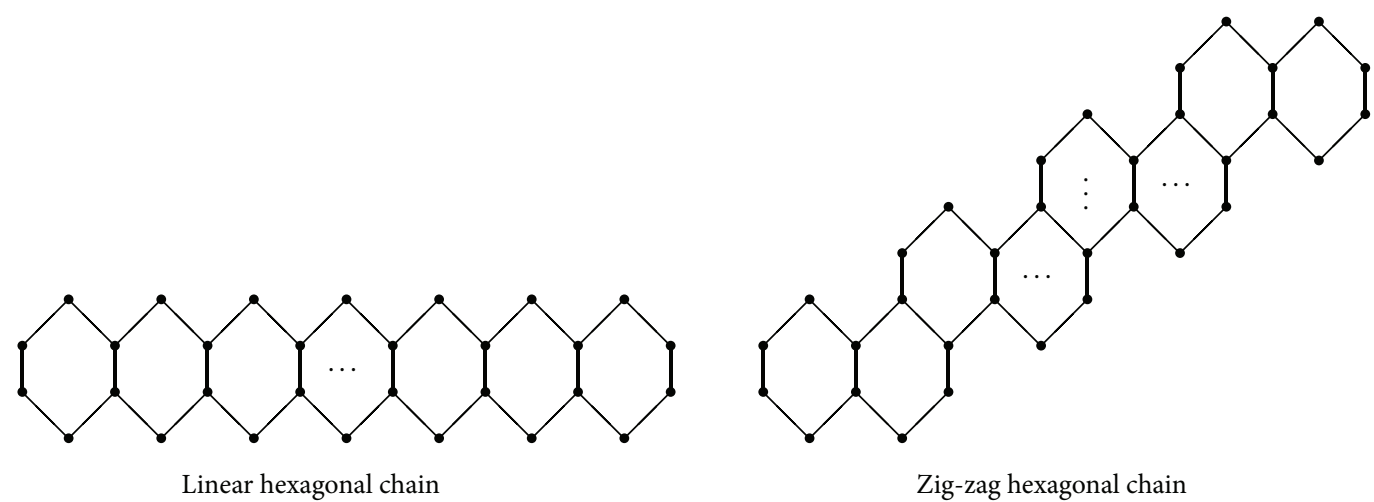

FIGURE 3: The structure of $L_{n}^{6}$ and $Z_{n}^{6}$.

\section{Second Atom-Bond Connectivity Index of Hexagonal Chain}

Hexagonal chain is one class of hexagonal system consisting of hexagonal. In hexagonal chain, each of two hexagonal has one common edge or no common vertex. Two hexagonal are adjacent if they have common edge. No three or more hexagonal share one vertex. Each hexagonal has two adjacent hexagonal except hexagonal in terminus, and each hexagonal chain has two hexagonal in terminus.

It is easy to verify that the hexagonal chain with $n$ hexagonal has $4 n+2$ vertices and $5 n+1$ edges. Let $L_{n}^{6}$ and $Z_{n}^{6}$ be the linear hexagonal chain and zig-zag hexagonal chain, respectively. For the chemical structure of $L_{n}^{6}$ and $Z_{n}^{6}$, one can refer to Figure 3 for more detail.

Again, we use the similar trick which was presented in Gutman and Klavžar [14], and we define elementary cut as follows. Choose an edge $e$ of the hexagonal system and draw a straight line through the center of $e$, orthogonal on $e$. This line will intersect the perimeter in two end points $P_{1}$ and $P_{2}$. The straight line segment $C$ whose end points are $P_{1}$ and $P_{2}$ is the elementary cut, intersecting the edge $e$. A fragment $S$ in hexagonal chain is just maximal linear chain which includes the hexagonal in start and end vertices. Let $l^{\prime}(S)$ be the length of fragment which denotes the number of hexagonal contained. Let $H_{n}^{6}$ be a hexagonal chain with $n$ hexagonal consisting of fragment sequence $S_{1}, S_{2}, \ldots, S_{m}(m \geq 1)$. Denote $l^{\prime}\left(S_{i}\right)=l_{i}^{\prime}(i=1, \ldots, m)$. Then, we verify that $l_{1}^{\prime}+l_{2}^{\prime}+$ $\cdots+l_{m}^{\prime}=n+m-1$ since each two adjacent fragments have one common hexagonal. For the $k$ th fragment of hexagonal chain, the cut of this fragment is the cut which intersects with $l_{k}^{\prime}+1$ parallel edges of hexagonal in this fragment. A fragment is called horizontal fragment if its cut parallels the horizontal direction; otherwise, it is called inclined fragment. Unilateral hexagonal chain is a special class of hexagonal chain such that the cut for each inclined fragment at the same angle with a horizontal direction. As an example, Figure 4 shows a structure of unilateral hexagonal chain. Clearly, linear hexagonal chain $L_{n}^{6}$ is a unilateral hexagonal chain with one fragment, and zig-zag is a unilateral hexagonal chain with $n-1$ fragments.

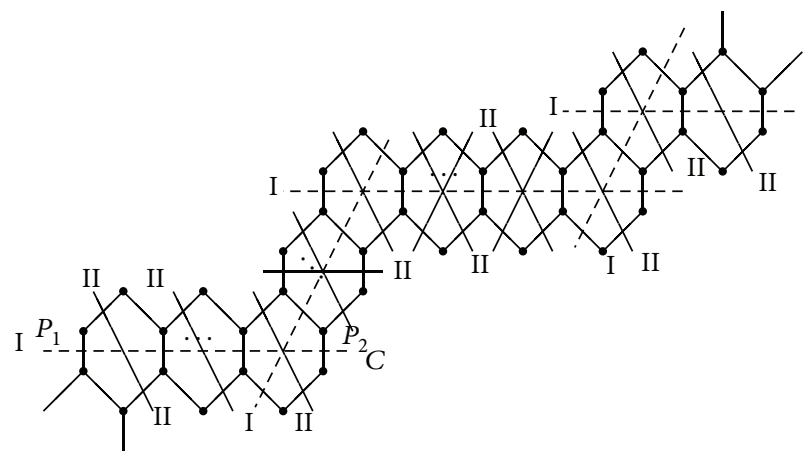

FIgURE 4: I-type cut and II-type cut of unilateral hexagonal chain.

We now show the second atom-bond connectivity index of unilateral hexagonal chain.

Theorem 4. Let $H_{n}^{6}$ be a unilateral hexagonal chain consisting of $m$ fragment $S_{1}, S_{2}, \ldots, S_{m}(m \geq 1)$, and let $l^{\prime}\left(S_{i}\right)=l_{i}^{\prime}(i=$ $1, \ldots, m)$ be the length of each fragment. Then, one has

$$
\begin{gathered}
A B C_{2}\left(H_{n}^{6}\right) \\
=4 \sum_{j=1}^{l_{1}^{\prime}-1} \sqrt{\frac{4 n}{(4 j-1)\left[4\left(\sum_{i=2}^{m} l_{i}^{\prime}-m+1\right)+4 l_{1}^{\prime}-4 j+3\right]}} \\
+2 \sum_{k=1}^{m-1}\left(4 n \left(\left[4\left(\sum_{i=1}^{k} l_{i}^{\prime}-k+1\right)-1\right]\right.\right. \\
\left.\left.\times\left[4\left(\sum_{i=k+1}^{m} l_{i}^{\prime}-m+k\right)+3\right]\right)^{-1}\right)^{1 / 2} \\
\times 4 \sum_{k=2}^{m-1} \sum_{j=2}^{\prime}\left(4 n\left(4\left(\sum_{i=1}^{k-1} l_{i}^{\prime}-k+1\right)+4 j-1\right]\right. \\
\left.\times\left[\sum_{i=k+1}^{m} l_{i}^{\prime}-m+k\right)^{-1}\right)^{1 / 2}
\end{gathered}
$$




$$
\begin{aligned}
&+4 \sum_{j=2}^{l_{m}^{\prime}}\left(4 n \left(\left[4\left(\sum_{i=1}^{m-1} l_{i}^{\prime}-m+1\right)+4 j-1\right]\right.\right.)^{-1}\right)^{1 / 2} \\
&\left.\left.\left.\times\left(4 l_{m}^{\prime}-4 j+3\right)\right)^{-1}\right)+2 l_{k}^{\prime}+1\right] \\
&+\sum_{k=2}^{m-1}\left(l_{k}^{\prime}+1\right) \\
& \times\left(4 n \left(4\left(\sum_{i=1}^{k-1} l_{i}^{\prime}-k+1\right)\right.\right. \\
&\left.\left.\times\left[4\left(\sum_{i=k+1}^{m} l_{i}^{\prime}-m+k\right)+2 l_{k}^{\prime}+1\right]\right)^{-1}\right)^{1 / 2} .
\end{aligned}
$$

Proof. The cuts in $H_{n}^{6}$ are divided into two types: I-type and II-type (see Figure 4). An edge is called I-type if it intersects with I-type cut. Also, an edge is called II-type if it intersects with II-type cut. In what follows, we consider two situations according to the type of an edge.

Case 1 . If edge $e$ is I-type in $j$ th square of $k$ th fragment (i.e., $e$ is edge which is passed by dotted line in Figure 4).

Subcase 1.1. If $k=1$, then we have

$$
\begin{gathered}
n_{1}(e)=2 l_{1}^{\prime}+1, \\
n_{2}(e)=4 \sum_{i=2}^{m}\left(l_{i}^{\prime}-m+1\right)+2 l_{1}^{\prime}+1 .
\end{gathered}
$$

Subcase 1.2. If $k=m$, then, we obtain

$$
\begin{gathered}
n_{1}(e)=4 \sum_{i=1}^{m-1}\left(l_{i}^{\prime}-m+1\right)+2 l_{k}^{\prime}+1, \\
n_{2}(e)=2 l_{m}^{\prime}+1 .
\end{gathered}
$$

Subcase 1.3. If $2 \leq k \leq m-1$, then we get

$$
\begin{aligned}
& n_{1}(e)=4 \sum_{i=1}^{k-1}\left(l_{i}^{\prime}-k+1\right)+2 l_{k}^{\prime}+1, \\
& n_{2}(e)=4 \sum_{i=k+1}^{m}\left(l_{i}^{\prime}-m+k\right)+2 l_{k}^{\prime}+1 .
\end{aligned}
$$

Case 2. If edge $e$ is II-type in $j$ th square of $k$ th fragment (i.e., $e$ is the edge which is passed by real line in Figure 4).

Subcase 2.1. If $k=1$, then we yield

$$
\begin{gathered}
n_{1}(e)=4 j-1, \\
n_{2}(e)=4 \sum_{i=2}^{m} l_{i}^{\prime}+4 l_{1}^{\prime}-4 j+3 .
\end{gathered}
$$

Subcase 2.2. If $k=m$, then we infer

$$
\begin{gathered}
n_{1}(e)=4 \sum_{i=1}^{m-1}\left(l_{i}^{\prime}-k+1\right)+4 j-1, \\
n_{2}(e)=4 l_{m}^{\prime}-4 j+3 .
\end{gathered}
$$

Subcase 2.3. If $2 \leq k \leq m-1$, then we deduce

$$
\begin{gathered}
n_{1}(e)=4 \sum_{i=1}^{k-1}\left(l_{i}^{\prime}-k+1\right)+4 j-1, \\
n_{2}(e)=4 \sum_{i=k+1}^{m} l_{i}^{\prime}+4\left(l_{k}^{\prime}-j\right)+3 .
\end{gathered}
$$

In particular, if $j=l_{k}^{\prime}$ in Subcase 2.3, we have $n_{1}(e)=$ $4 \sum_{i=1}^{k}\left(l_{i}^{\prime}-k+1\right)-1$ and $n_{2}(e)=4 \sum_{i=k+1}^{m}\left(l_{i}^{\prime}-m+k\right)+3$.

Hence, we get the desired result by combining the above cases and the definition of the second atom-bond connectivity index.

Corollary 5. Let $L_{n}^{6}$ be the linear chain with $n$ hexagonal. Then, one has

$$
A B C_{2}\left(L_{n}^{6}\right)=4 \sum_{j=1}^{n-1} \sqrt{\frac{4 n}{(4 j-1)(4 n-4 j+3)}} .
$$

Proof. By the definition of linear chain, we check that $m=$ $1, l_{1}^{\prime}=n, l_{2}^{\prime}=\cdots=l_{m}^{\prime}=0$. In terms of Theorem 4 , we shortly get the result.

Corollary 6. Let $Z_{n}^{6}$ be the zig-zag chain with $n$ hexagonal. Then, one has

$$
\begin{aligned}
A B C_{2}\left(Z_{n}^{6}\right)= & 8 \sqrt{\frac{4 n}{3(4 n-1)}} \\
& +2 \sum_{k=1}^{n-2} \sqrt{\frac{4 n}{(4 k+3)(4 n+4 k-1)}} \\
& +3 \sum_{k=2}^{n-2} \sqrt{\frac{4 n}{(4 k+1)(4 n-4 k+1)}} .
\end{aligned}
$$

Proof. Using the definition of zig-zag chain, we verify that $m=n-1$ and $l_{1}^{\prime}=l_{2}^{\prime}=\cdots=l_{m}^{\prime}=2$. In view of Theorem 4, the corollary is immediately yielded.

\section{Second Atom-Bond Connectivity Index of V-Phenylenic Nanotubes and Nanotori}

The notations in this section follow from Diudea [15]. In what follows, we use $n(u)$ and $n(v)$ to express $n_{1}(e)$ and $n_{2}(e)$ for short, respectively. The molecular structures $\mathrm{V}$-phenylenic nanotube and $\mathrm{V}$-phenylenic nanotorus are denoted by $V P H X[m, n]$ and $V P H Y[m, n]$, respectively.

The next result shows the representation of $A B C_{2}(V P H X[m, n])$, and the structure of $\operatorname{VPHX}[m, n]$ is described in Figure 5. 


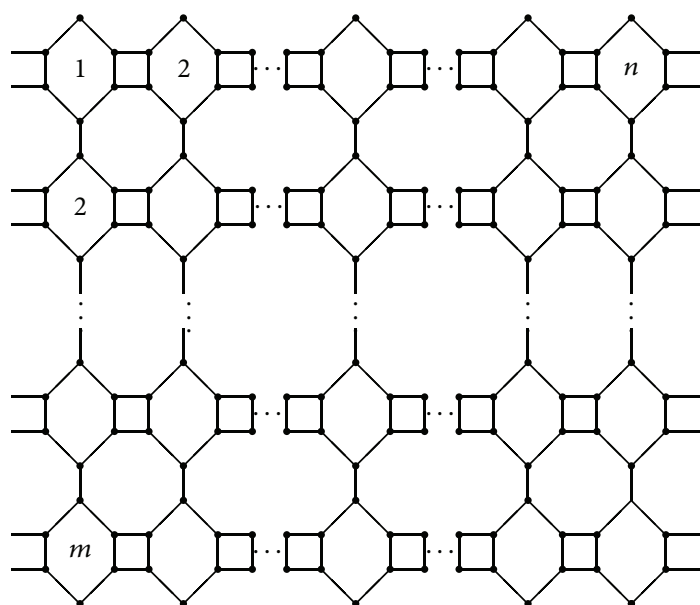

FIGURE 5: The structure of V-phenylenic nanotube.

Theorem 7. Consider

$$
A B C_{2}(V P H X[m, n])= \begin{cases}\alpha_{1}, & \text { if } m \neq n \\ \alpha_{2}, & \text { if } m=n\end{cases}
$$

where $\alpha_{1}$ and $\alpha_{2}$ are presented in the process of the proof.

Proof. First, we note that $|V(\operatorname{VPHX}[m, n])|=6 m n$. To compute the second atom-bond connectivity index of $V P H X[m, n]$, we assume that $E_{1}, E_{2}$, and $E_{3}$ are the set of all vertical, oblique, and horizontal edges, respectively. Then, we infer

$$
\begin{aligned}
A C_{2} & (V P H X[m, n]) \\
= & \sum_{e \in E(V P H X[m, n])} \sqrt{\frac{n(u)+n(v)-2}{n(u) n(v)}} \\
= & \sum_{e \in E_{1}} \sqrt{\frac{n(u)+n(v)-2}{n(u) n(v)}} \\
& +\sum_{e \in E_{2}} \sqrt{\frac{n(u)+n(v)-2}{n(u) n(v)}} \\
& +\sum_{e \in E_{3}} \sqrt{\frac{n(u)+n(v)-2}{n(u) n(v)}} \\
= & 2 \sum_{i=1}^{n-1} \sqrt{\frac{(6 m i)+(6 m n-6 m i)(2 m)-2}{(6 m i)(6 m n-6 m i)(2 m)}} \\
& +\sum_{i=1}^{n-1} \sqrt{\frac{(3 m i)+(6 m n-3 m i)(2 n)-2}{(3 m i)(6 m n-3 m i)(2 n)}} \\
& +\sum_{e \in E_{3}} \sqrt{\frac{n(u)+n(v)-2}{n(u) n(v)} .}
\end{aligned}
$$

Now, we calculate $\sum_{e \in E_{3}} \sqrt{(n(u)+n(v)-2) / n(u) n(v)}$ by considering the following two cases.
Case $1(m \neq n)$. Under this situation, we deduce

$$
\begin{aligned}
& \sum_{e \in E_{3}} \sqrt{\frac{n(u)+n(v)-2}{n(u) n(v)}} \\
&=2 \sum_{i=1}^{|m-n|-1}\left(\left((2 \beta)\left(S_{\beta}+(6 \beta-3) i\right)\right.\right. \\
&\left.+\left(6 m n-S_{\beta}-(6 \beta-3) i\right)-2\right) \\
& \times\left((2 \beta)\left(S_{\beta}+(6 \beta-3) i\right)\right. \\
&\left.\left.\times\left(6 m n-S_{\beta}-(6 \beta-3) i\right)\right)^{-1}\right)^{1 / 2},
\end{aligned}
$$

where $S_{i}=3+9+15+\cdots+(6 i-3)$ and $\beta=\min \{m, n\}$. Hence, in this case, we infer that the second atom-bond connectivity index of $V P H X[m, n]$ is

$$
\begin{gathered}
\alpha_{1}=2 \sum_{i=1}^{n-1} \sqrt{\frac{(6 m i)+(6 m n-6 m i)(2 m)-2}{(6 m i)(6 m n-6 m i)(2 m)}} \\
+\sum_{i=1}^{n-1} \sqrt{\frac{(3 m i)+(6 m n-3 m i)(2 n)-2}{(3 m i)(6 m n-3 m i)(2 n)}} \\
+2 \sum_{i=1}^{|m-n|-1}\left(\left((2 \beta)\left(S_{\beta}+(6 \beta-3) i\right)\right.\right. \\
\left.+\left(6 m n-S_{\beta}-(6 \beta-3) i\right)-2\right) \\
\times\left((2 \beta)\left(S_{\beta}+(6 \beta-3) i\right)\right. \\
\left.\left.\times\left(6 m n-S_{\beta}-(6 \beta-3) i\right)\right)^{-1}\right)^{1 / 2}
\end{gathered}
$$

Case $2(m=n)$. In this case, we get

$$
\begin{aligned}
\sum_{e \in E_{3}} & \sqrt{\frac{n(u)+n(v)-2}{n(u) n(v)}} \\
& =\sqrt{\frac{4 n\left(S_{n}+6 n-3\right)+\left(6 m n-S_{n}-(6 n-3)\right)-2}{4 n\left(S_{n}+6 n-3\right)\left(6 m n-S_{n}-(6 n-3)\right)}} .
\end{aligned}
$$

Thus, the corresponding second atom-bond connectivity index is

$$
\begin{aligned}
\alpha_{2}= & 2 \sum_{i=1}^{n-1} \sqrt{\frac{(6 m i)+(6 m n-6 m i)(2 m)-2}{(6 m i)(6 m n-6 m i)(2 m)}} \\
& +\sum_{i=1}^{n-1} \sqrt{\frac{(3 m i)+(6 m n-3 m i)(2 n)-2}{(3 m i)(6 m n-3 m i)(2 n)}} \\
& +\sqrt{\frac{4 n\left(S_{n}+6 n-3\right)+\left(6 m n-S_{n}-(6 n-3)\right)-2}{4 n\left(S_{n}+6 n-3\right)\left(6 m n-S_{n}-(6 n-3)\right)}} .
\end{aligned}
$$

The result is archived. 


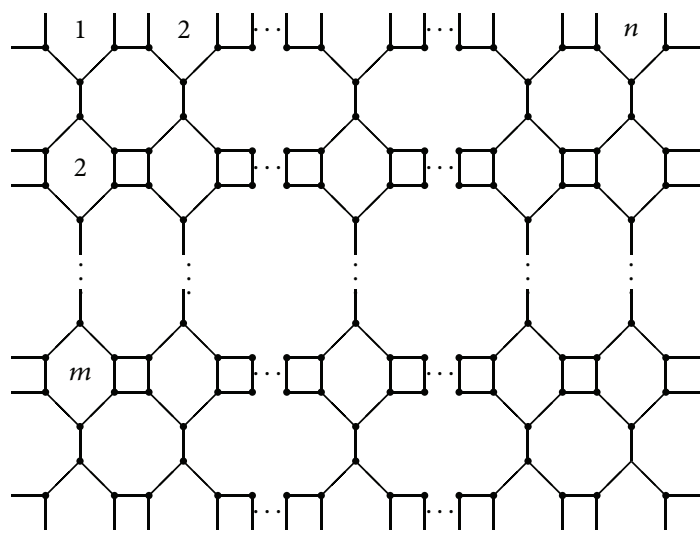

FIGURE 6: The structure of V-phenylenic nanotorus.

Our last result computes the second atom-bond connectivity index of $V P H Y[m, n]$ and the structure of $V P H Y[m, n]$ presented in Figure 6.

Theorem 8. Consider

$$
A B C_{2}(V P H Y[m, n])= \begin{cases}\alpha_{3}, & \text { if } m \neq n \\ \alpha_{4}, & \text { if } m=n,\end{cases}
$$

where $\alpha_{3}$ and $\alpha_{4}$ are presented in the process of the proof.

Proof. In order to prove the theorem, we use a similar trick as in Theorem 7. Obviously, $|V(V P H Y[m, n])|=6 m n$. We assume that $E_{1}, E_{2}$, and $E_{3}$ are the set of all vertical, oblique, and horizontal edges, respectively. Then we deduce

$$
\begin{aligned}
A B C_{2} & (V P H Y[m, n]) \\
= & \sum_{e \in E(V P H Y[m, n])} \sqrt{\frac{n(u)+n(v)-2}{n(u) n(v)}} \\
= & \sum_{e \in E_{1}} \sqrt{\frac{n(u)+n(v)-2}{n(u) n(v)}} \\
& +\sum_{e \in E_{2}} \sqrt{\frac{n(u)+n(v)-2}{n(u) n(v)}} \\
& +\sum_{e \in E_{3}} \sqrt{\frac{n(u)+n(v)-2}{n(u) n(v)}} \\
= & 2 \sum_{i=1}^{n-1} \sqrt{\frac{(6 m i)+(6 m n-6 m i)(2 m)-2}{(6 m i)(6 m n-6 m i)(2 m)}} \\
& +2 \sum_{i=1}^{n-1} \sqrt{\frac{(3 m i)+(6 m n-3 m i)(2 n)-2}{(3 m i)(6 m n-3 m i)(2 n)}} \\
& +\sum_{e \in E_{3}} \sqrt{\frac{n(u)+n(v)-2}{n(u) n(v)} .}
\end{aligned}
$$

Now, we calculate $\sum_{e \in E_{3}} \sqrt{(n(u)+n(v)-2) / n(u) n(v)}$ according to the relationship between $m$ and $n$.

Case $1(m \neq n)$. Under this assumption, we infer

$$
\begin{aligned}
& \sum_{e \in E_{3}} \sqrt{\frac{n(u)+n(v)-2}{n(u) n(v)}} \\
&=4 \sum_{i=1}^{|m-n|-1}\left(\left((2 \beta)\left(S_{\beta}+(6 \beta-3) i\right)\right.\right. \\
&\left.+\left(6 m n-S_{\beta}-(6 \beta-3) i\right)-2\right) \\
& \times\left((2 \beta)\left(S_{\beta}+(6 \beta-3) i\right)\right. \\
&\left.\left.\times\left(6 m n-S_{\beta}-(6 \beta-3) i\right)\right)^{-1}\right)^{1 / 2},
\end{aligned}
$$

where $S_{i}=3+9+15+\cdots+(6 i-3)$ and $\beta=\min \{m, n\}$. Hence, the second atom-bond connectivity index of $V P H Y[m, n]$ for $m \neq n$ is

$$
\begin{gathered}
\alpha_{3}=2 \sum_{i=1}^{n-1} \sqrt{\frac{(6 m i)+(6 m n-6 m i)(2 m)-2}{(6 m i)(6 m n-6 m i)(2 m)}} \\
+2 \sum_{i=1}^{n-1} \sqrt{\frac{(3 m i)+(6 m n-3 m i)(2 n)-2}{(3 m i)(6 m n-3 m i)(2 n)}} \\
+4 \sum_{i=1}^{|m-n|-1}\left(\left((2 \beta)\left(S_{\beta}+(6 \beta-3) i\right)\right.\right. \\
\left.+\left(6 m n-S_{\beta}-(6 \beta-3) i\right)-2\right) \\
\times\left((2 \beta)\left(S_{\beta}+(6 \beta-3) i\right)\right. \\
\left.\left.\times\left(6 m n-S_{\beta}-(6 \beta-3) i\right)\right)^{-1}\right)^{1 / 2}
\end{gathered}
$$

Case $2(m=n)$. In this case, we have

$$
\begin{aligned}
\sum_{e \in E_{3}} \sqrt{\frac{n(u)+n(v)-2}{n(u) n(v)}} \\
=\sqrt{\frac{4 n\left(S_{n}+6 n-3\right)+\left(6 m n-S_{n}-(6 n-3)\right)-2}{4 n\left(S_{n}+6 n-3\right)\left(6 m n-S_{n}-(6 n-3)\right)}} .
\end{aligned}
$$


Thus, the corresponding second atom-bond connectivity index is

$$
\begin{aligned}
\alpha_{4}= & 2 \sum_{i=1}^{n-1} \sqrt{\frac{(6 m i)+(6 m n-6 m i)(2 m)-2}{(6 m i)(6 m n-6 m i)(2 m)}} \\
& +2 \sum_{i=1}^{n-1} \sqrt{\frac{(3 m i)+(6 m n-3 m i)(2 n)-2}{(3 m i)(6 m n-3 m i)(2 n)}} \\
& +\sqrt{\frac{4 n\left(S_{n}+6 n-3\right)+\left(6 m n-S_{n}-(6 n-3)\right)-2}{4 n\left(S_{n}+6 n-3\right)\left(6 m n-S_{n}-(6 n-3)\right)}} .
\end{aligned}
$$

The proof is completed.

\section{Conflict of Interests}

The authors declare that there is no conflict of interests regarding the publication of this paper.

\section{Acknowledgments}

The authors thank the reviewers for their constructive comments in improving the quality of this paper. They also would like to thank the anonymous referees for providing them with constructive comments and suggestions. The research is partially supported by NSFC (nos. 11401519, 11371328, and 11471293).

\section{References}

[1] L. Yan, Y. Li, W. Gao, and J. Li, "PI index for some special graphs," Journal of Chemical and Pharmaceutical Research, vol. 5, no. 11, pp. 260-264, 2013.

[2] L. Yan, Y. Li, W. Gao, and J. Li, “On the extremal hyper-wiener index of graphs," Journal of Chemical and Pharmaceutical Research, vol. 6, no. 3, pp. 477-481, 2014.

[3] W. Gao and L. Shi, "Wiener index of gear fan graph and gear wheel graph," Asian Journal of Chemistry, vol. 26, no. 11, pp. 3397-3400, 2014.

[4] W. F. Xi and W. Gao, "Geometric-arithmetic index and Zagreb indices of certain special molecular graphs," Journal of Advances in Chemistry, vol. 10, no. 2, pp. 2254-2261, 2014.

[5] J. Y. Dou, Y. Y. Wang, and W. Gao, "Some characteristics on hyper-wiener index of graphs," Journal of Chemical and Pharmaceutical Research, vol. 6, no. 5, pp. 1659-1663, 2014.

[6] J. A. Bondy and U. S. R. Murty, Graph Theory, Springer, Berlin, Germany, 2008.

[7] E. Estrada, L. Torres, L. Rodríguez, and I. Gutman, "An atombond connectivity index: modelling the enthalpy of formation of alkanes," Indian Journal of Chemistry A, vol. 37, no. 10, pp. 849-855, 1998.

[8] K. C. Das, I. Gutman, and B. Furtula, "On atom-bond connectivity index," Chemical Physics Letters, vol. 511, pp. 452-454, 2011.

[9] B. Furtula, A. Graovac, and D. Vukičević, "Atom-bond connectivity index of trees," Discrete Applied Mathematics, vol. 157, no. 13, pp. 2828-2835, 2009.
[10] T. S. Vassilev and L. J. Huntington, "On the minimum $A B C$ index of chemical trees," Applied Mathematics, vol. 2, no. 1, pp. 8-16, 2012.

[11] J. Chen, J. Liu, and Q. Li, "The atom-bond connectivity index of catacondensed polyomino graphs," Discrete Dynamics in Nature and Society, vol. 2013, Article ID 598517, 7 pages, 2013.

[12] A. Graovac and M. Ghorbani, "A new version of atom-bond connectivity index," Acta Chimica Slovenica, vol. 57, no. 3, pp. 609-612, 2010.

[13] M. Rostami, M. S. Haghighat, and M. Ghorbani, "On second atom-bond connectivity index," Iranian Journal of Mathematical Chemistry, vol. 4, no. 2, pp. 265-270, 2013.

[14] I. Gutman and S. Klavžar, "An algorithm for the calculation of the Szeged index of benzenoid hydrocarbons," Journal of Chemical Information and Computer Sciences, vol. 35, no. 6, pp. 1011-1014, 1995.

[15] M. V. Diudea, "Phenylenic and naphthylenic tori," Fullerenes, Nanotubes and Carbon Nanostructures, vol. 10, no. 4, pp. 273$292,2002$. 

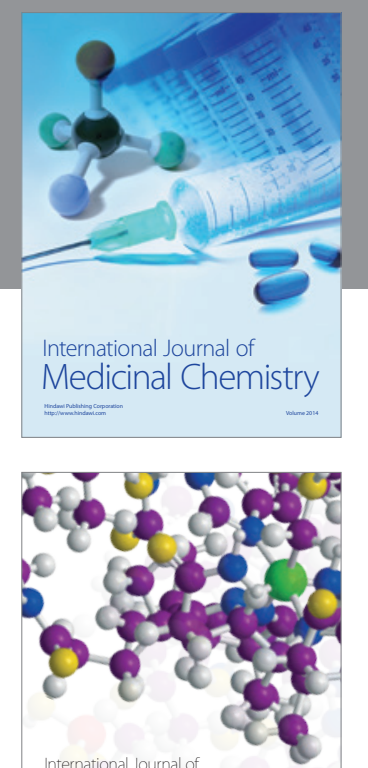

\section{Carbohydrate} Chemistry



The Scientific World Journal
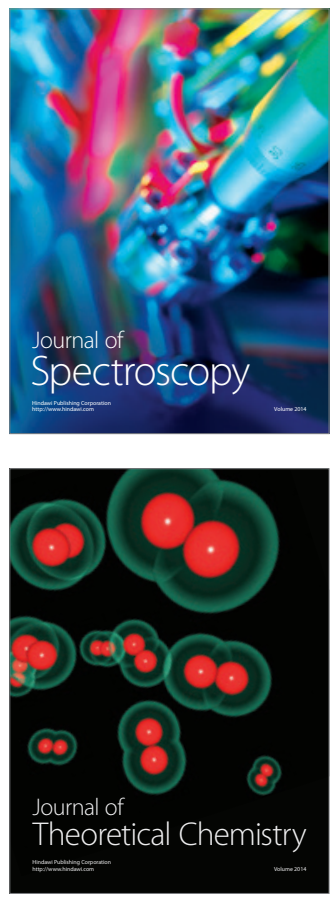
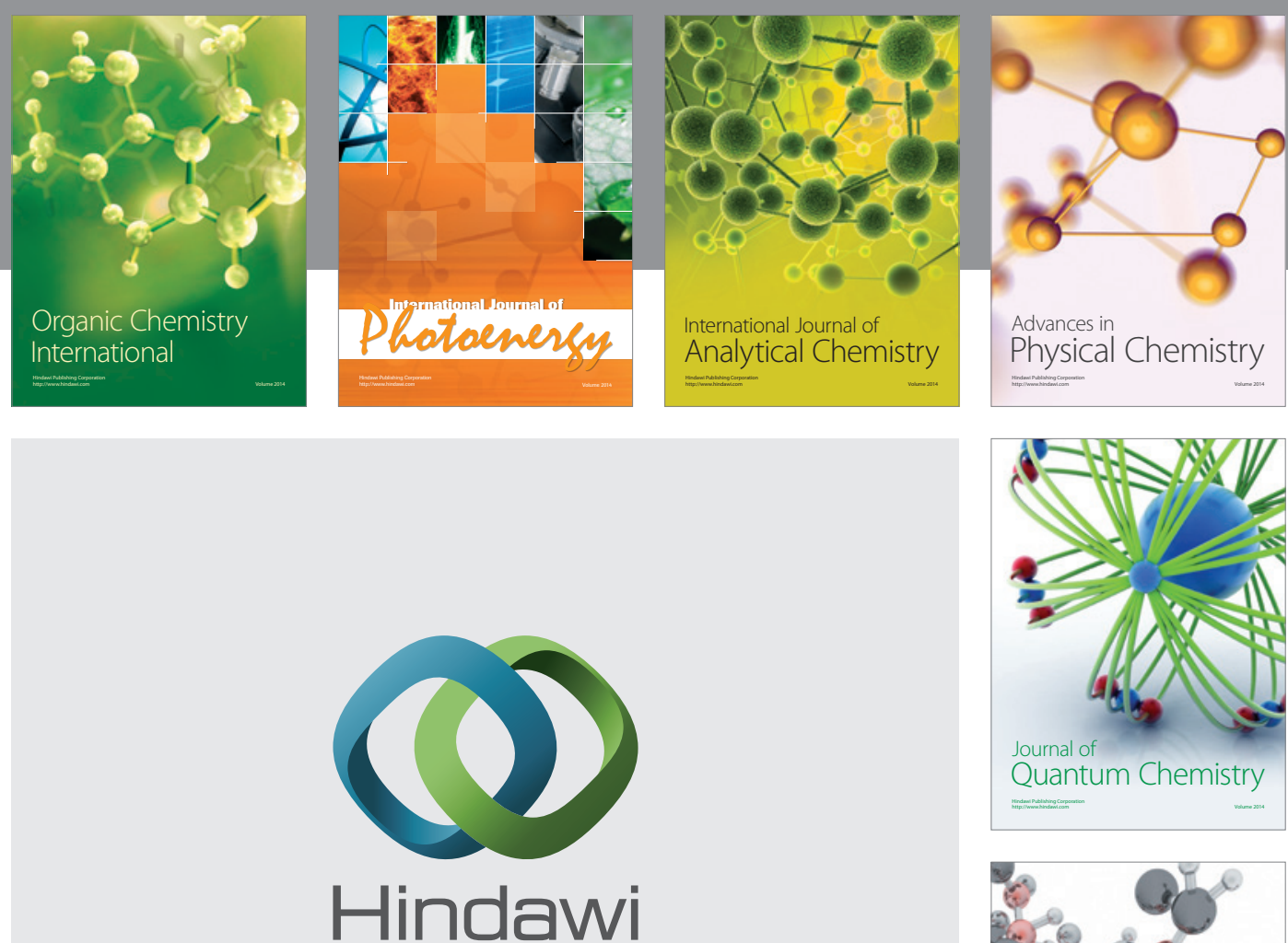

Submit your manuscripts at

http://www.hindawi.com

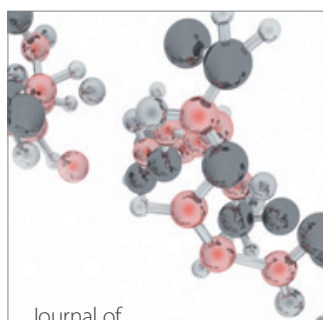

Analytical Methods

in Chemistry

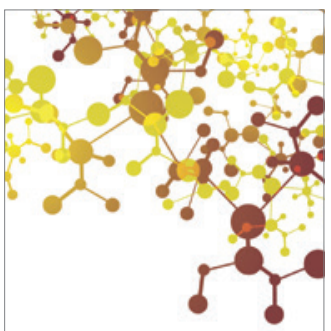

Journal of

Applied Chemistry

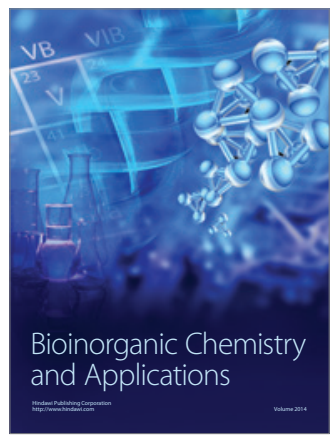

Inorganic Chemistry
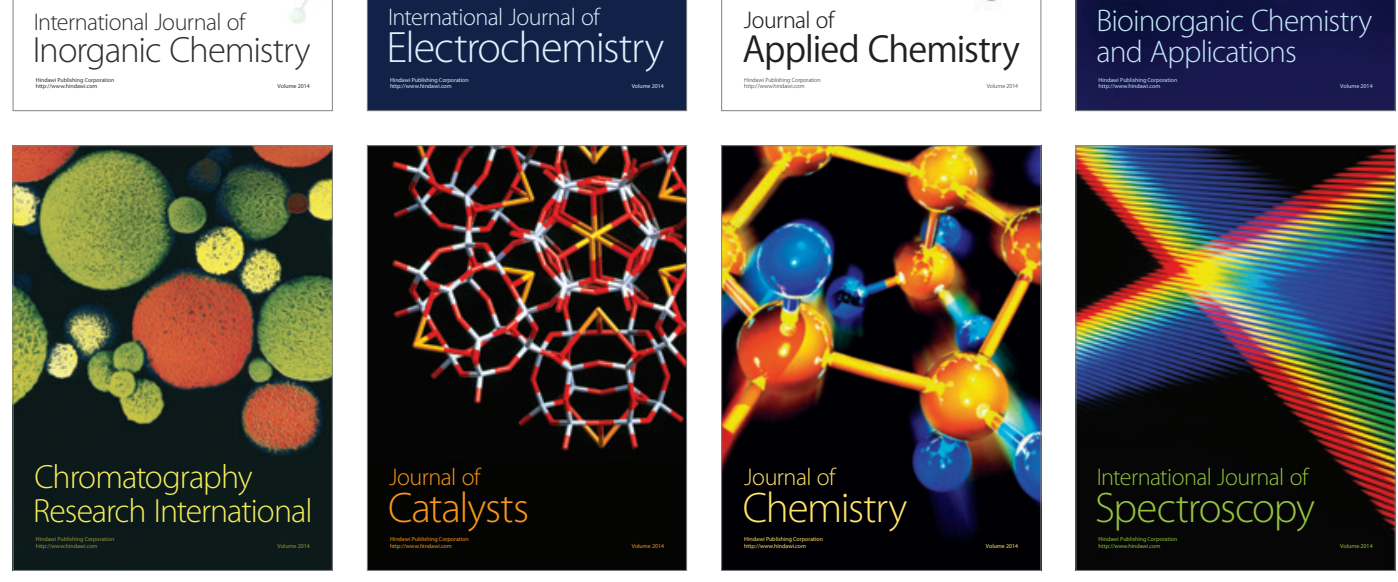